\title{
Transcranial Magnetic Stimulation Disrupts the Perception and Embodiment of Facial Expressions
}

\author{
David Pitcher, Lúcia Garrido, Vincent Walsh, and Bradley C. Duchaine \\ Institute of Cognitive Neuroscience and Department of Psychology, University College London, London WC1N 3AR, United Kingdom
}

\begin{abstract}
Theories of embodied cognition propose that recognizing facial expressions requires visual processing followed by simulation of the somatovisceral responses associated with the perceived expression. To test this proposal, we targeted the right occipital face area (rOFA) and the face region of right somatosensory cortex (rSC) with repetitive transcranial magnetic stimulation (rTMS) while participants discriminated facial expressions. rTMS selectively impaired discrimination of facial expressions at both sites but had no effect on a matched face identity task. Site specificity within the rSC was demonstrated by targeting rTMS at the face and finger regions while participants performed the expression discrimination task. rTMS targeted at the face region impaired task performance relative to rTMS targeted at the finger region. To establish the temporal course of visual and somatosensory contributions to expression processing, double-pulse TMS was delivered at different times to rOFA and rSC during expression discrimination. Accuracy dropped when pulses were delivered at $60-100 \mathrm{~ms}$ at rOFA and at 100-140 and 130-170 $\mathrm{ms}$ at rSC. These sequential impairments at rOFA and rSC support embodied accounts of expression recognition as well as hierarchical models of face processing. The results also demonstrate that nonvisual cortical areas contribute during early stages of expression processing.
\end{abstract}

Key words: face perception; embodied cognition; emotion; transcranial magnetic stimulation; somatosensory cortex; occipital face area

\section{Introduction}

Neurobiological models of face processing propose that faceselective areas in the inferior occipital gyrus represent facial information before analysis in downstream areas in the fusiform gyrus and superior temporal sulcus (Haxby et al., 2000; Calder and Young, 2005). The involvement of these face-selective areas in facial expression recognition finds support from neuroimaging and neuropsychological patient studies (Rossion et al., 2003; Winston et al., 2003; Steeves et al., 2006; Engell and Haxby, 2007).

Theories of embodied cognition, however, propose that visual mechanisms alone are insufficient and that a nonvisual process of internally simulating the somatovisceral and motor responses associated with the observed emotion is also necessary for expression recognition (Carr et al., 2003; Niedenthal, 2007). This hypothesis leads to the prediction that expression recognition can be disrupted by interference with the simulation process. Behavioral and physiological evidence supports this prediction. Expression recognition is impaired by facial contortions that restrict the capacity to produce expressions (Oberman et al., 2007) and by somatovisceral responses evoked by unpleasant tastes and smells (Wicker et al., 2003; Jabbi et al., 2007). Expression-relevant facial muscles exhibit increased electromyographic responses to subliminal exposure to emotional expressions (Dimberg et al., 2000).

\footnotetext{
Received April 4, 2008; revised June 30, 2008; accepted July 2, 2008.

This work was supported by Biotechnology and Biological Sciences Research Council Grant BB/F022875/1 (B.C.D., V.W.). We thank Neil Muggleton, Michael Banissy, Gill Rhodes, and Andy Calder.

Correspondence should be addressed to David Pitcher, Institute of Cognitive Neuroscience and Department of Psychology, University College London, Alexandra House, 17 Queen Square, London WC1N 3AR, UK. E-mail: d.pitcher@ucl.ac.uk.

DOI:10.1523/JNEUROSCI.1450-08.2008

Copyright $\odot 2008$ Society for Neuroscience $\quad$ 0270-6474/08/288929-05\$15.00/0
}

A meta-analysis of patients with focal brain lesions reported that damage to right somatosensory cortices was associated with expression recognition impairments (Adolphs et al., 2000), and a functional magnetic resonance imaging (fMRI) study also demonstrated that the right somatosensory cortex (rSC) shows an increased response when participants discriminate between facial expressions (Winston et al., 2003) (but see Hariri et al., 2000; Andrews and Ewbank, 2004).

To assess the embodied cognition account of expression recognition, we delivered repetitive transcranial magnetic stimulation (rTMS) over the right occipital face area (rOFA) in the inferior occipital gyrus or the face area of rSC while participants matched either facial expressions or facial identities. The rOFA exhibits a stronger response to faces than to other categories (Gauthier et al., 2000) and is the first stage in hierarchical faceprocessing models (Haxby et al., 2000; Calder and Young, 2005; Fairhall and Ishai, 2007). The somatosensory cortex is the sensory-receptive area for representations of the body and has a disproportionately large region dedicated to the face (Penfield and Jasper, 1954; Huang and Sereno, 2007). Although embodied cognition accounts propose that the somatosensory cortex plays a general role in expression recognition, a TMS study using happy and fearful faces reported that rSC stimulation only impaired discrimination of fearful faces (Pourtois et al., 2004). To further test the embodied cognition account, we used six expressions and stimulated rOFA and two somatosensory regions.

\section{Materials and Methods}

Participants. Twenty-eight right-handed participants with normal or corrected-to-normal vision (13 males, 15 females, aged 19-41 years) gave informed consent as directed by University College London ethics 
committee. Twelve participated in experiment 1 , eight in experiment 2, and 14 in experiment 3. Six participants took part in both experiments 1 and 3. One participant withdrew during experiment 1 because of discomfort with TMS stimulation.

Materials. Stimuli were presented centrally on a super video graphics array 17 inch monitor (resolution, $1024 \times 768$; refresh rate, $100 \mathrm{~Hz}$ ). Stimuli were six female models (C, MF, MO, NR, PF, and SW) from Ekman and Friesen's (1976) facial affect series expressing one of six emotions: happy, sad, surprise, fear, disgust, and anger. Each grayscale picture was cropped with the same contour using Adobe Photoshop to cover the hair and neck. The same set of faces was used for both the identity and expression discrimination blocks.

For the expression discrimination task, half the trials showed picture pairs with the same expression and half showed pairs with different expressions. Identity always changed between sample and target faces. The six expressions were presented an equal number of times.

For the identity discrimination task, half the trials showed pairs with the same identity and half showed pairs with different identities. Expression always changed between the sample and target faces. The six models were presented an equal number of times.

TMS stimulation and site localization. TMS was delivered at $10 \mathrm{~Hz}$ and $60 \%$ of maximal stimulator output, using a Magstim Super Rapid Stimulator and a $70 \mathrm{~mm}$ figure-of-eight coil, with the coil handle pointing upward and parallel to the midline. A single intensity was used on the basis of previous studies (O'Shea et al., 2004; Pitcher et al., 2007), and, for ease of comparison with related studies, the majority of which have used a single intensity. Because we used within-site task controls, any task-specific effects could not be explained by induced TMS intensity differences within participants.

In blocks with TMS during experiment 1 and experiment 2, test stimuli were presented during 500 ms rTMS with rTMS onset concurrent with the onset of the target visual stimulus. During experiment 3, double-pulse TMS (dTMS) with $40 \mathrm{~ms}$ between pulses (O'Shea et al., 2004; Pitcher et al., 2007) was delivered at seven different times from stimulus onset: 20 $60,60-100,100-140,130-170,170-210,210-$

250 , and $250-290 \mathrm{~ms}$, chosen to cover the most likely times of rOFA and rSC involvement (Pourtois et al., 2004; Pitcher et al., 2007).

Each participant's MRI structural scan was normalized against a standard template, and each transformation (FSL software; Oxford University Centre for Functional MRI of the Brain) was used to convert the appropriate Talairach coordinates to the untransformed (structural) space coordinates, yielding subject-specific localization of the sites (Fig. 1) (supplemental Fig. 1, available at www.jneurosci.org as supplemental material). The Talairach coordinates for $\operatorname{rOFA}(38,-80,-7)$ were the average from 11 neurologically normal participants in an fMRI face processing study (Rossion et al., 2003), and coordinates for the face region of $\operatorname{rSC}(44,-12,48)$ were the average from 12 neurologically normal participants in an fMRI study of facial expression (Winston et al., 2003). The Talairach coordinates for the finger region of $\operatorname{rSC}(47,-30,62)$ were the average for six neurologically normal participants in an fMRI cortical mapping study (Huang and Sereno, 2007). The vertex was defined as a point midway between the inion and the nasion and equidistant from the

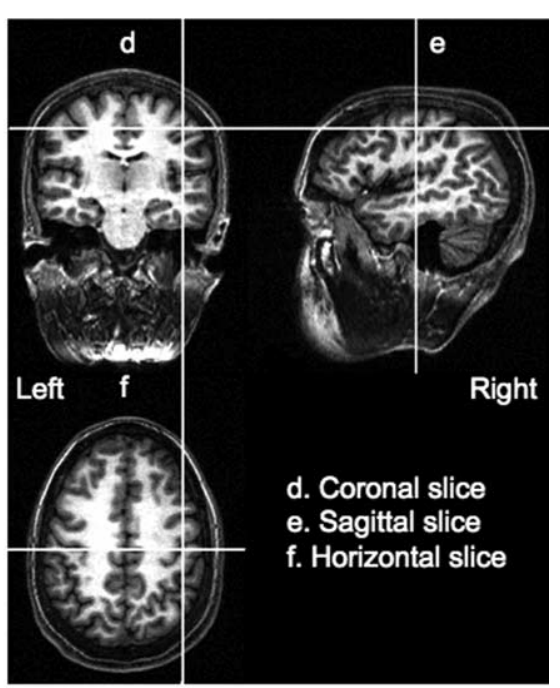

Normalized location of the face region of right somatosensory cortex in one subject. Based on Talairach coordinates $44,-12,48$ a. Coronal slice

b. Sagittal slice

c. Horizontal slice

Normalized location of the right occipital face (rOFA) in one subject. Based on

Figure 1. The normalized location of the rOFA and the face region of the rSC in one subject.

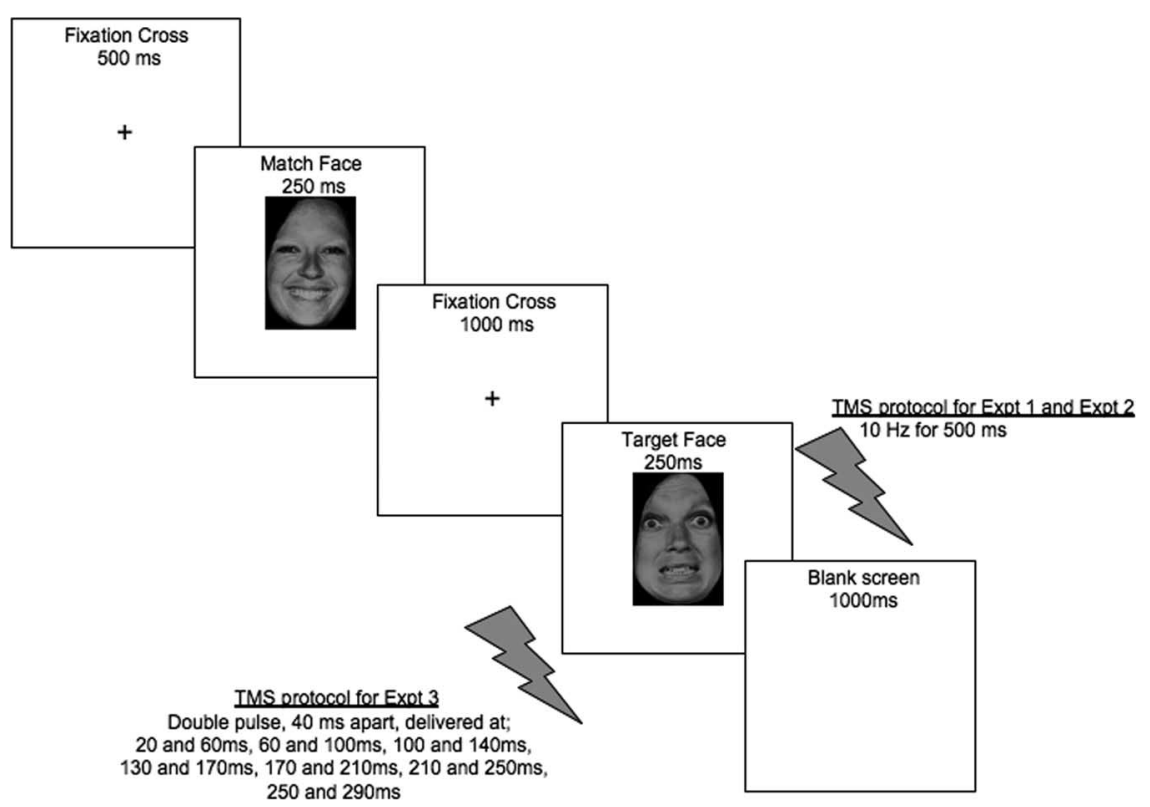

Figure 2. Timeline of the trial procedure for experiments 1-3.

left and right intertragal notches. Using these coordinates, TMS sites were located using the Brainsight TMS-MRI coregistration system (Rogue Research).

Procedure. Experiment 1 delivered rTMS to rOFA, the face region of rSC, and vertex during the behavioral tasks. The vertex condition served as a control for nonspecific effects of TMS. A no-TMS condition was included as a behavioral baseline. The identity component acted as a control task based on the results of a pilot experiment (supplemental Figs. 2, 3, available at www.jneurosci.org as supplemental material).

Figure 2 displays the trial procedure. Participants sat $57 \mathrm{~cm}$ from the monitor with their heads stabilized in a chin rest and indicated by a right-hand key press whether the prime face showed the same facial expression as the target face (expression task) or the same person as the target face (identity task). They were instructed to respond as accurately and quickly as possible.

Four blocks of 72 trials were presented for each task (expression and identity), and task order was balanced between participants. During each 


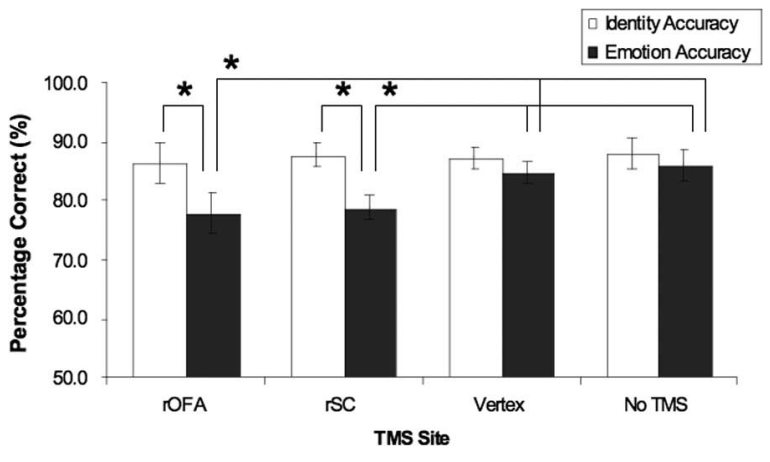

Figure 3. Mean accuracy scores for the expression and identity discrimination tasks in experiment 1. An asterisk denotes a significant difference in Bonferroni's corrected tests.

task rTMS was delivered to rOFA, the face region of rSC and vertex, a no-TMS block was also included. Block order was balanced between participants. Within each block, the trial order was randomized.

Experiment 2 required participants to perform only the expression matching task while rTMS was targeted at the face or finger region of rSC or at vertex. Site stimulation order was balanced between participants. Each block consisted of 72 trials.

Experiment 3 required participants to perform only the expression task. It was done in two testing sessions on different days, one session stimulated rOFA and vertex, whereas the other stimulated the face region of rSC and vertex. Session order was balanced between participants, and both sessions were completed within $7 \mathrm{~d}$ for all participants. There were 36 trials per timing condition block. Timing condition order and TMS stimulation site were balanced among participants.

\section{Results}

The aim of experiment 1 was to interfere with the participants' ability to match different facial expressions by delivering rTMS over rOFA and the face region of rSC. The vertex was also stimulated as an active TMS control site, and a no-TMS condition was included for comparison. The stimulated regions were identified on each participant's structural MRI scan and coregistered with the coil position using neuronavigation methods. The effects on mean accuracy performance in the expression task and the face identity control task are shown in Figure 3. The main finding was that both rOFA and rSC stimulation reduced participants' accuracy on the expression task only. There was no effect on the identity task.

A two $X$ four-way repeated-measures ANOVA of the accuracy results showed a main effect of TMS site $\left(F_{(3,33)}=10.3 ; p>\right.$ $0.001)$ and of expression versus identity $\left(F_{(1,11)}=10.6 ; p=\right.$ $0.008)$. TMS site and expression versus identity also combined in a two-way interaction $\left(F_{(3,33)}=4.3 ; p=0.012\right)$. Bonferroni's corrected post hoc comparisons showed significant impairments for the expression task relative to the identity task when stimulating $\mathrm{rOFA}(p=0.017)$ and $\mathrm{rSC}(p<0.001)$. More importantly, accuracy on the expression discrimination task was significantly impaired by rTMS to rOFA relative to vertex $(p=0.008)$ and no TMS $(p=0.007)$. Similarly, there were significant impairments at $\mathrm{rSC}$ relative to vertex $(p=0.004)$ and no TMS $(p=0.010)$. There was no significant difference between the expression and identity tasks when rTMS was targeted at vertex or when no TMS was delivered.

A two $X$ four-way ANOVA on the reaction time (RT) data showed a significant slowing of RTs on expression trials compared with identity $\left(F_{(1,11)}=6.3 ; p=0.029\right)$ but no two-way interaction between TMS site and expression versus identity
$\left(F_{(3,33)}=1.571 ; p=0.215\right)$ (supplemental Fig. 4, available at www.jneurosci.org as supplemental material).

This first experiment thus established that rOFA and the face region of rSC are important for expression discrimination. To preclude an account of the data based on differential difficulty of the identity and expression tasks, four paired sample $t$ tests were performed. These showed significant RT differences at rOFA $(t=$ $3.25 ; p=0.008)$ and $\mathrm{rSC}(t=4.56 ; p=0.001)$ but not at vertex $(t=1.63 ; p=0.132)$ or the no-TMS condition $(t=0.64 ; p=$ $0.534)$.

Because another study of the rSC (Pourtois et al., 2004) found TMS effects for fearful but not for happy faces, we examined our data for expression-specific effects. We categorized trials according to the expression in the second stimulus and analyzed the error scores. A four $\times$ six-way repeated-measures ANOVA showed a main effect of TMS site $\left(F_{(3,33)}=6.9 ; p=0.001\right)$ but not of expression $\left(F_{(5,55)}=1.4 ; p=0.24\right)$, and the interaction did not approach significance $\left(F_{(15,165)}=1.4 ; p=0.54\right)$.

\section{Spatial specificity of the TMS effect in rSC}

Although experiment 1 demonstrated site- and task-specific effects, the possibility remained that our stimulation of rSC was not specific to the face area, which is close to the regions representing other body parts such as the fingers (Penfield and Jasper, 1954; Huang and Sereno, 2007). We therefore targeted rTMS at the face region and the finger region in experiment 2 to assess whether we could dissociate the expression effects in these areas. Again we used vertex as an active TMS control site. Mean accuracy scores revealed a spatially specific effect limited to the face region of rSC. rTMS at the face area reduced accuracy to $79.00 \%$ compared with accuracy of $83.25 \%$ at the finger area and $84.13 \%$ at vertex. A one $X$ three-way repeated-measures ANOVA showed a main effect of TMS site $\left(F_{(1,7)}=12.8 ; p=0.009\right)$. Bonferroni's corrected post hoc comparisons revealed a significant performance difference between the face region and the finger region ( $p=$ $0.021)$ and the face region and vertex $(p=0.027)$. There was no significant difference between the finger region and vertex ( $p=$ 0.787).

A four $\times$ six-way repeated-measures ANOVA was performed to test for expression-specific effects. It showed no main effects of TMS site $\left(F_{(2,14)}=2.9 ; p=0.093\right)$ or expression $\left(F_{(5,35)}=1.3\right.$; $p=0.28)$, and the interaction did not approach significance $\left(F_{(10,70)}=1.5 ; p=0.16\right)$.

\section{Temporal specificity of TMS effects in rOFA and rSC}

In the first two experiments, we demonstrated that the TMS effects on expressions were specific to rOFA and to the face region of rSC. To better understand the roles of these areas in expression processing, it is necessary to be able to comment on the timing of the involvement of each area. If, as hypothesized, the two areas are sequential components in a distributed hierarchical network (Haxby et al., 2000; Adolphs, 2002), then TMS-induced interference at rOFA should precede TMS interference at rSC. We therefore stimulated rOFA, the face region of rSC, and vertex using dTMS with $40 \mathrm{~ms}$ between pulses. The dTMS pulses were delivered at seven different times from stimulus onset in pairs at 20 $60,60-100,100-140,130-170,170-210,210-250$, and 250-290 $\mathrm{ms}$. Previous studies suggested that this $270 \mathrm{~ms}$ period would encompass the involvement of both rOFA and SC in expression processing (Pourtois et al., 2004; Pitcher et al., 2007). As Figure 4 shows, accuracy was selectively and significantly reduced when dTMS was delivered over rOFA in a pulse pair delivered at $60-$ $100 \mathrm{~ms}$ after stimulus onset. In contrast, when dTMS was deliv- 
ered over the face region of $\mathrm{rSC}$, it selectively impaired expression accuracy at two later time windows, $100-140$ and 130-170 ms after stimulus onset.

To make the above statistical comparison, we first established that the vertex control site showed no significant differences between the two testing sessions. A two $\times$ seven repeated-measures ANOVA for the accuracy data showed no main effect of either TMS site $\left(F_{(1,14)}=1.7 ; p=\right.$ $0.2)$ or timing $\left(F_{(6,84)}=0.2 ; p=0.9\right)$ and no interaction $\left(F_{(6,84)}=0.8 ; p=0.6\right)$. A two $X$ seven ANOVA for the RT data also showed no main effect of TMS site $\left(F_{(1,14)}\right.$ $=1 ; p=0.8)$ or timing $\left(F_{(6,84)}=1.5 ; p=\right.$ $0.2)$ and no interaction $\left(F_{(6,84)}=1 ; p=\right.$ $0.4)$. Therefore, to simplify additional analysis, we collapsed the two vertex blocks together by taking mean scores at all timing conditions for the accuracy and RT data.

A three $X$ seven-way repeated-measures ANOVA showed a main effect of timing $\left(F_{(6,84)}=3 ; p=0.01\right)$ but not of TMS site $\left(F_{(2,28)}=2.6 ; p=0.09\right)$. TMS site and timing combined in a significant two-way interaction $\left(F_{(12,168)}=4 ; p<0.001\right)$. Bonferroni's corrected post hoc tests showed that, when dTMS was applied in a 60-100 ms pair, there was a significant difference between rOFA and vertex $(p<0.001)$ and between rOFA and rSC $(p=0.008)$. The temporally specific effect on rSC was later. dTMS over the face region of rSC significantly reduced accuracy on the expression task compared with stimulation at the vertex control site when delivered in pulse-pair timings at $100-140 \mathrm{~ms}$ $(p=0.01)$ and $130-170 \mathrm{~ms}(p=0.018)$.

A three $\times$ seven-way repeated-measures ANOVA on the RT data showed no significant effects (supplemental Fig. 5, available at www.jneurosci.org as supplemental material).

\section{Discussion}

The results demonstrate that facial expression matching is dependent on both the rOFA and the rSC. As an embodied cognition account predicts, facial expression recognition is not solely a visual task. Behavioral studies have shown that contortions of the face disrupt expression recognition (Oberman et al., 2007), and TMS targeted at rSC may act in a manner analogous to the contortions by disrupting the somatic simulation of a perceived expression. The results of experiment 2 also support the embodied cognition hypothesis by demonstrating that rTMS targeted at the face region of rSC impaired expression discrimination relative to rTMS targeted at the finger region and the vertex.

An analysis of the expression task errors in experiments 1 and 2 failed to demonstrate preferential impairment of specific expressions by rTMS to rSC. This contrasts with a study that reported an impairment of fearful faces compared with happy faces (Pourtois et al., 2004). We used six expressions from Ekman and Friesen's (1976) set that restricted the number of trials per expression, so it is possible that lack of statistical power accounts for the lack of an expression-specific effect.

The sequential impairments observed at rOFA and rSC in experiment 3 support existing face processing models (Haxby et al., 2000; Adolphs, 2002). The 60-100 ms impairment at rOFA demonstrates that rOFA processes expression information at an early stage in the face processing stream and replicates the timing of rOFA TMS effects in a face part discrimination task (Pitcher et al., 2007). Impairment at rSC encompassed two time windows,
$100-140$ and $130-170 \mathrm{~ms}$, and indicates that the area is active over a comparatively longer time period than rOFA. This suggests that embodying an expression in the rSC is a sustained process relative to the visual process at rOFA. The timing of the $\mathrm{rSC}$ effect suggests that the contribution from nonvisual cortical areas to expression discrimination may co-occur with visually mediated face computations such as those producing the face-selective N170 component in evoked response potential studies (Bentin et al., 1996). The timing of this contribution is also consistent with studies that have reported that cortical areas outside the visual system exhibit a response earlier than the N170 in visual tasks involving facial expressions (Eimer and Holmes, 2002) and emotionally evocative images (Kawasaki et al., 2001).

Because our matching task required comparison of two sequentially presented expressions, one account of the right somatosensory disruption in experiments 1 and 2 predicts that it resulted from disruption of a frontoparietal working memory (WM) network (Harris et al., 2001; Oliveri et al., 2001; Mottaghy et al., 2002). However, if this were the case, the identity task should have been as impaired as the expression task. Also, in experiment 2, there was no impairment on the expression task when rTMS was targeted at the finger region of rSC, as would be expected if a WM network was disrupted.

The absence of an effect on identity at rOFA is interesting given the structure of face processing models (Haxby et al., 2000; Calder and Young, 2005). These models propose that identity and expression processing depend on separate mechanisms in later stages of the face processing stream but that both are processed in rOFA. Support for rOFA involvement in identity computations comes from fMRI adaptation studies (Yovel and Kanwisher, 2004) and from patient studies (Rossion et al., 2003). However, our previous TMS study at rOFA found an effect only for face part discrimination and not for face part spacing discrimination (Pitcher et al., 2007). In the current experiment, the two faces presented on each trial in the identity task always differed in their expressions and hence the shape of their face parts. As a result, TMS may not have affected identity processing because participants were forced to rely on information other than the face parts (e.g., relative spacing, surface reflectance). Furthermore, Rotshtein et al. (2005) have shown that OFA adapts to physical changes in faces even when the identity remained unchanged, whereas the fusiform gyrus only showed release from adaptation when facial identity changed. This suggests that rTMS to rOFA might not have affected performance in our identity task because it required discrimination between identities and not 
physical changes within an identity. It is also possible that some routes to facial identity do not require OFA; Rotshtein et al. (2007), for example, showed that the middle occipital gyrus transmits low spatial frequency identity information to the fusiform gyrus. Neuropsychological results also suggest that early visual areas are directly connected to the fusiform gyrus (Sorger et al., 2007).

\section{References}

Adolphs R (2002) Neural systems for recognizing emotion. Curr Opin Neurobiol 12:169-177.

Adolphs R, Damasio H, Tranel D, Cooper G, Damasio AR (2000) A role for somatosensory cortices in the visual recognition of emotion as revealed by three-dimensional lesion mapping. J Neurosci 20:2683-2690.

Andrews TJ, Ewbank MP (2004) Distinct representations for facial identity and changeable aspects of faces in human visual cortex. Neuroimage 23:905-913.

Bentin S, Allison T, Puce A, Perez E, McCarthy G (1996) Electrophysiological studies of face perception in humans. J Cogn Neurosci 8:551-565.

Calder AJ, Young AW (2005) Understanding the recognition of facial identity and facial expression. Nat Rev Neurosci 6:641-651.

Carr L, Iacoboni M, Dubeau M, Mazziotta J, Lenzi GL (2003) Neural mechanisms of empathy in humans: a relay from neural systems for imitation to limbic areas. Proc Natl Acad Sci U S A 100:5497-5502.

Dimberg U, Thunberg M, Elmehed K (2000) Unconscious facial reactions to emotional facial expressions. Psych Science 11:86-89.

Eimer M, Holmes A (2002) An ERP study on the time course of emotional face processing. Neuroreport 13:427-431.

Ekman P, Friesen WV (1976) Pictures of facial affect. Palo Alto, CA: Consulting Psychologists.

Engell AD, Haxby JV (2007) Facial expression and gaze-direction in human superior temporal sulcus. Neuropsychologia 45:3234-3241.

Fairhall SL, Ishai A (2007) Effective connectivity within the distributed cortical network for face perception. Cereb Cortex 17:2400-2406.

Gauthier I, Tarr MJ, Moylan J, Skudlarski P, Gore JC, Anderson AW (2000) The fusiform "face area" is part of a network that processes faces at the individual level. J Cogn Neurosci 12:495-504.

Hariri AR, Bookheimer SY, Mazziotta JC (2000) Modulating emotional responses: effects of a neocortical network on the limbic system. Neuroreport 11:43-48.

Harris JA, Harris IM, Diamond ME (2001) The topography of tactile working memory. J Neurosci 21:8262-8269.

Haxby JV, Hoffman EA, Gobbini MI (2000) The distributed human neural system for face perception. Trends Cogn Sci 4:223-233.

Huang RS, Sereno MI (2007) Dodecapus: an MR-compatible system for somatosensory stimulation. Neuroimage 34:1060-1073.

Jabbi M, Swart M, Keysers C (2007) Empathy for positive and negative emotions in the gustatory. cortex. Neuroimage 34:1744-1753.
Kawasaki H, Kaufman O, Damasio H, Damasio AR, Granner M, Bakken H, Hori T, Howard MA III, Adolphs R (2001) Single-neuron responses to emotional visual stimuli recorded in human ventral prefrontal cortex. Nat Neurosci 4:15-16.

Mottaghy FM, Gangitano M, Sparing R, Krause BJ, Pascual-Leone A (2002) Segregation of areas related to visual working memory in the prefrontal cortex revealed by rTMS. Cereb Cortex 12:369-375.

Niedenthal PM (2007) Embodying emotion. Science 316:1002-1005.

O'Shea J, Muggleton NG, Cowey A, Walsh V (2004) Timing of target discrimination in human frontal eye fields. J Cogn Neurosci 16:1060-1067.

Oberman LM, Winkielman P, Ramachandran VS (2007) Face to face. Soc Neurosci 2:167-178.

Oliveri M, Turriziani P, Carlesimo GA, Koch G, Tomaiuolo F, Panella M, Caltagirone C (2001) Parieto-frontal interactions in visual-object and visual-spatial working memory. Cereb Cortex 11:606-618.

Penfield W, Jasper H (1954) Epilepsy and the functional anatomy of the human brain. Boston: Little, Brown.

Pitcher D, Walsh V, Yovel G, Duchaine B (2007) TMS evidence for the involvement of the right occipital face area in early face processing. Curr Biol 17:1568-1573.

Pourtois G, Sander D, Andres M, Grandjean D, Reveret L, Olivier E, Vuilleumier P (2004) Dissociable roles of the human somatosensory and superior temporal cortices for processing social face signals. Eur J Neurosci 20:3507-3515.

Rossion B, Caldara R, Seghier M, Schuller AM, Lazeyras F, Mayer E (2003) A network of occipito-temporal face-sensitive areas besides the right middle fusiform gyrus is necessary for normal face processing. Brain 126:2381-2395.

Rotshtein P, Henson RN, Treves A, Driver J, Dolan RJ (2005) Morphing Marilyn into Maggie dissociates physical and identity face representations in the brain. Nat Neurosci 8:107-113.

Rotshtein P, Vuilleumier P, Winston J, Driver J, Dolan R (2007) Distinct and convergent visual processing of high and low spatial frequency information in faces. Cereb Cortex 17:2713-2724.

Sorger B, Goebel R, Schiltz C, Rossion B (2007) Understanding the functional neuroanatomy of prosopagnosia. Neuroimage 35:836-852.

Steeves JK, Culham JC, Duchaine BC, Pratesi CC, Valyear KF, Schindler I, Humphrey GK, Milner AD, Goodale MA (2006) The fusiform face area is not sufficient for face recognition. Neuropsychologia 44:594-609.

Wicker B, Keysers C, Plailly J, Royet JP, Gallese V, Rizzolatti G (2003) Both of us disgusted in my insula: the common neural basis of seeing and feeling disgust. Neuron 40:655-664.

Winston JS, O'Doherty J, Dolan RJ (2003) Common and distinct neural responses during direct and incidental processing of multiple facial emotions. Neuroimage 20:84-97.

Yovel G, Kanwisher N (2004) Face perception: domain specific, not process specific. Neuron 44:889-898. 